\section{Research Square}

Preprints are preliminary reports that have not undergone peer review. They should not be considered conclusive, used to inform clinical practice, or referenced by the media as validated information.

\title{
Early Initiation of Breastfeeding up to Six Months and Breastfeeding Experience of Mothers who had Cesarean Section: A Scoping Review
}

Yunefit Ulfa ( $\nabla$ 17dn901@slcn.ac.jp)

St. Luke's international University Japan https://orcid.org/0000-0001-5728-337X

Naoko MARUYAMA

St. Luke's School of Nursing: Saint Luke's School of Nursing

Yumiko IGARASHI

St. Luke's School of Nursing: Saint Luke's School of Nursing

Shigeko HORIUCHI

St. Luke's School of Nursing: Saint Luke's School of Nursing

\section{Research}

Keywords: breast feeding, cesarean section, lactation, postpartum period

Posted Date: December 29th, 2021

DOI: https://doi.org/10.21203/rs.3.rs-1157298/v1

License: (c) (i) This work is licensed under a Creative Commons Attribution 4.0 International License. Read Full License 


\section{Abstract}

Background: Breastfeeding is a gold nutritional feeding for the infant for optimal growth and development. Early initiation of breastfeeding is an important initial step for successful continuing breastfeeding. Cesarean section (C-section) has been reported to have negative effects on early initiation of breastfeeding. However, no current literature summarized the breastfeeding rate after C-section and vaginal delivery globally. Therefore, this scoping review aimed to systematically collect, assess, and map the existing literature regarding the rate and experience of breastfeeding of mothers after $\mathrm{C}$-section.

Design: We conducted a scoping review in accordance with the PRISMA extension for scoping reviews (PRISMA-ScR) statement.

Methods: We performed an electronic database search on Cumulative Index of Nursing and Allied Health Literature, PubMed, Embase, Cochrane Library, and PsychINFO on March 16, 2021. The inclusion criteria were (a) research, experiential, and case reports; (b) reports on the rate of breastfeeding after Csection and vaginal delivery; (c) qualitative studies on the experience of breastfeeding after C-section.

Results: The search identified 4635 potentially relevant articles. After screening, 27 articles (24 quantitative and three qualitative studies) were included in the scoping review from 1990 to 2020 . Most studies reported a higher breastfeeding rate in mothers who had vaginal delivery than in mothers who had CSection at breastfeeding initiation, hospital discharge, one month, three months, and six months postoperatively. A large difference in breastfeeding rate was found at early breastfeeding initiation between the vaginal delivery and C-section groups. Most studies showed a breastfeeding rate of more than $30 \%$ at late breastfeeding initiation, one month, and three months after C-section. A mother's physical discomfort, low self-efficacy, and lack of knowledge, and the insufficient support from healthcare providers were identified as breastfeeding barriers after C-section.

Conclusions: The rate of breastfeeding after C-section has remained low to date. Lack of breastfeeding knowledge and insufficient healthcare provider support after $\mathrm{C}$-section are the common underlying issues. Approaches to enhance breastfeeding must be developed and consistently implemented.

\section{Background}

Cesarean section (C-section) is an invasive delivery procedure performed via abdominal and uterine incisions to save the mother and baby from a high-risk vaginal delivery (VD) [1]. C-section has been increasing worldwide and has surpassed the percentage set by the World Health Organization (WHO) (i.e., $5 \%-15 \%$ ) [2]. Maternal request has been reported as one of the reasons for the increasing number of C-section [2.3]

C-section can be a necessary lifesaving procedure. However, performing C-section without any medical indication has been correlated with higher maternal mortality, and maternal and infant morbidity [4]. Moreover, C-section has been attributed to increased complications of subsequent deliveries and breastfeeding experience [2]. Previous studies have shown that women who had C-section are less likely to breastfeed and delay their breastfeeding initiation, which is an important predictor of continued breastfeeding [5-7]

Breastfeeding is considered to provide the best nutrition to infants and reduce infant morbidity significantly. The WHO recommends starting breastfeeding within one hour after delivery and to continue exclusive breastfeeding up to six months [8]. Globally, $44 \%$ of newborns are breastfed within the first hour after birth [9]. A population-based study in Ethiopia found that women undergoing a C-section had an $86 \%$ lower rate of early initiation of breastfeeding [10]. In Canada reported $4.3 \%$ women did not initiate breastfeeding after C-section[11]. Parity, type of delivery, time from birth to first contact with the mother, and socioeconomic status influence the success of breastfeeding [12]. The type of delivery has a considerable association with breastfeeding practices, and C-section is considered to have a negative effect on early breastfeeding initiation [6]. To the best of our knowledge, there has been no scoping review on the rate of breastfeeding from early initiation of breastfeeding up to 6 months after C-section, as well as the metasyntheses of qualitative studies on barriers to breastfeeding after C-section.

\section{Aims}

This scoping review aims to systematically collect, assess, and map the existing literature regarding the rate of breastfeeding from its early initiation up to 6 months after $\mathrm{C}$-section, as well as the breastfeeding experiences of mothers after C-section.

\section{Methods}

This scoping review used the methodological framework of Joanna Briggs Institutes (JBI) of 2017 and was conducted in accordance with the Preferred Reporting Items for Systematic reviews and Meta-Analyses extension for Scoping Reviews (PRISMA-ScR). The JBI framework comprises the following elements: identify relevant studies in accordance with the research objectives, select eligible studies, chart the data, summarise, and report the results [13].

\subsection{Eligibility criteria}

The inclusion criteria and exclusion criteria set for this study were (Table 1); 
Table 1

Study inclusion and exclusion criteria

\begin{tabular}{|ll}
\hline Inclusion criteria & Exclusion criteria \\
\hline Research, experiential, and case reports & Editorials, letters, commentaries, opinion papers, and grey literature studies \\
\hline Reports on the rate or percentage of breastfeeding & Report the odds ratio \\
$\begin{array}{l}\text { Reports on the rate or percentage of breastfeeding of both after C- } \\
\text { section and VD }\end{array}$ & $\begin{array}{l}\text { Reports on the rate or percentage of breastfeeding only after C-section or } \\
\text { only after VD }\end{array}$ \\
English & Non-English \\
\hline Full text & Non full text \\
\hline High and moderate quality (based on critical appraisal) & Poor quality (based on critical appraisal) \\
\hline
\end{tabular}

\subsection{Search strategy and information sources}

An electronic database literature search was conducted on March 16, 2021. The databases searched included Cumulative Index of Nursing and Allied Health Literature, PubMed, Embase, Cochrane Library, and PsychINFO. The search terms were related to postpartum, breastfeeding, and C-section. The full search strategy is shown in Appendix 1. Date limitation was not set.

\subsection{Selection of studies}

The search results were downloaded into RefWorks to identify and remove duplicates. The results were then transferred to Rayyan application to ease screening [14]. Titles and abstracts that met the inclusion criteria were independently screened by the lead researcher. Then, eligible full text articles were assessed independently for inclusion in the study.

Although quality appraisal is not required in scoping reviews, three researchers critically appraised the included studies using the JBI framework for crosssectional and cohort studies and the critical appraisal skill programme for qualitative studies to rigorously screen the studies included and remove poorquality studies. The quality score of the studies were divided into three categories: high quality (total score $>70 \%$ ), moderate quality (total score $40 \%-70 \%$ ), and poor quality (total score $<40 \%$ ). The three researchers scored the articles independently, and the scores were then compared and discussed. An old article published in 1990 was included in this study because of its valuable old study.

\subsection{Data charting}

Data were extracted by the lead researcher and confirmed by the other researchers. The extracted studies were characterized as follows: (1) author(s), (2) year of publication, (3) country, (4) study design, (5) objectives, (6) population, (7) Other factors associated with breast feeding initiation and exclusive breastfeeding, (8) summary of results, and (9) quality appraisal.

\section{Results}

\subsection{Selection of sources}

The database search identified 4635 potentially relevant articles. Of these, 1238 duplicate articles were removed. A total of 3397 articles were screened and 3332 records were excluded based on their titles and abstracts. The remaining 65 articles were assessed for eligibility. After full text screening, 38 articles that did not meet the eligibility criteria were removed. Finally, 27 articles were included in the scoping review (Figure 1).

Twenty-seven published articles from 1990-2020 were identified and included in this scoping review. Twenty-four were quantitative studies and three were qualitative studies. As for the quantitative studies, nine articles were cross-sectional studies and 15 articles were cohort studies.

\subsection{Study characteristics}

The studies were conducted in the following countries: Five studies from African continent [15-19], four studies from Canada [11,20-22], three studies from China [5,7,23], two studies from England [24,25] and two studies from Italy [12,26], and one studies from each of the following; Australia [27], Bangladesh [28], Denmark [29], Lebanon [30], Puerto Rico [31], Sweden [32], Saudi Arabia [33], Taiwan [34], Turkey [35], United State of America [36], and Vietnam [37].

The present study investigated the breastfeeding rate of mothers after C-section and VD. We included the time of breastfeeding. There were 13 studies on the early initiation of breastfeeding (within one hour after delivery), seven studies on the late initiation of breastfeeding (after one hour of delivery), six studies on breastfeeding at hospital discharge (hospitalization: two to five days), eight studies on exclusive breastfeeding one month after delivery, six studies on exclusive breastfeeding three months after delivery, and eight studies on exclusive breastfeeding six months after delivery.

\subsection{Synthesis of results}

The characteristics of included studies is summarized in Table 2. The results showed that most of mothers who had VD can initiate breastfeeding compared with mothers who had C-section. Most of the studies showed that a successful early initiation of breastfeeding will continue to exclusive breastfeeding at one and three months. However, we cannot definitively conclude an association of early initiation of breastfeeding with exclusive breastfeeding at six months. 
Table 2

Summary of results of included studies

\begin{tabular}{|c|c|c|c|c|c|c|c|c|c|}
\hline NO & Authors & Year & Country & Study Design & Objectives & Population & $\begin{array}{l}\text { Summary of } \\
\text { Results }\end{array}$ & $\begin{array}{l}\text { Other factors } \\
\text { associated } \\
\text { with breast } \\
\text { feeding } \\
\text { initiation and } \\
\text { exclusive } \\
\text { breastfeeding }\end{array}$ & $\begin{array}{l}\text { Summary } \\
\text { of Critical } \\
\text { Appraisal }\end{array}$ \\
\hline 1 & $\begin{array}{l}\text { Vestermark } \\
\text { et al.[29] }\end{array}$ & 1990 & Denmark & $\begin{array}{l}\text { Quantitative, } \\
\text { cohort study }\end{array}$ & $\begin{array}{l}\text { To evaluate } \\
\text { whether the } \\
\text { mode of } \\
\text { delivery affects } \\
\text { breastfeeding. }\end{array}$ & $\begin{array}{l}\text { Cesarean } \\
\text { section } \\
(C S)=102 \\
\text { Vaginal } \\
\text { delivery } \\
(\mathrm{VD})=231\end{array}$ & $\begin{array}{l}\text { - Mothers who } \\
\text { gave birth by CS } \\
\text { had a delay in } \\
\text { their early } \\
\text { initiation of } \\
\text { breastfeeding. } \\
\text { - Their babies } \\
\text { were prescribed } \\
\text { formula milk } \\
\text { - Postdelivery } \\
\text { formula milk or } \\
\text { prelacteal feeding } \\
\text { had no effect on } \\
\text { exclusive } \\
\text { breastfeeding one } \\
\text { month after } \\
\text { delivery. }\end{array}$ & & $\begin{array}{l}\text { Poor } \\
\text { quality }\end{array}$ \\
\hline 2 & $\begin{array}{l}\text { Wiklund, } \\
\text { Edman, \& } \\
\text { Andolf [32] }\end{array}$ & 2007 & Sweden & $\begin{array}{l}\text { Quantitative, } \\
\text { cohort study }\end{array}$ & $\begin{array}{l}\text { To investigate } \\
\text { the } \\
\text { breastfeeding } \\
\text { of mothers } \\
\text { who } \\
\text { experienced CS } \\
\text { for the first } \\
\text { time }\end{array}$ & $\begin{array}{l}C S=357 \\
V D=266\end{array}$ & $\begin{array}{l}\text { - Many mothers } \\
\text { who gave birth by } \\
\text { CS had weaned } \\
\text { their babies three } \\
\text { months after birth. } \\
\text { - The reason for } \\
\text { this was related to } \\
\text { personality traits } \\
\text { and } \\
\text { sociodemographic } \\
\text { factors, not to the } \\
\text { type of delivery. }\end{array}$ & & $\begin{array}{l}\text { Moderate } \\
\text { quality }\end{array}$ \\
\hline 3 & $\begin{array}{l}\text { Chien, L. - } \\
\text { \& Tai [34] }\end{array}$ & 2007 & Taiwan & $\begin{array}{l}\text { Quantitative, } \\
\text { cohort study }\end{array}$ & $\begin{array}{l}\text { To evaluate the } \\
\text { effects of type } \\
\text { of delivery on } \\
\text { the initiation of } \\
\text { breastfeeding, } \\
\text { and } \\
\text { breastfeeding } \\
\text { at } 1 \text { and } 3 \\
\text { months after } \\
\text { delivery. }\end{array}$ & $\begin{array}{l}C S=699 \\
V D=1124\end{array}$ & $\begin{array}{l}\text { - Breastfeeding } \\
\text { initiation within } 30 \\
\text { minutes after } \\
\text { delivery was } \\
\text { associated with a } \\
\text { higher chance of } \\
\text { breastfeeding at } \\
\text { one and three } \\
\text { months after } \\
\text { delivery. }\end{array}$ & $\begin{array}{l}\text { breastfeeding } \\
\text { initiation: } \\
\text { maternal age, } \\
\text { education, } \\
\text { work status, } \\
\text { and spousal } \\
\text { sup- } \\
\text { port for } \\
\text { breastfeeding. }\end{array}$ & $\begin{array}{l}\text { Moderate } \\
\text { quality }\end{array}$ \\
\hline 4 & $\begin{array}{l}\text { Pérez-Ríos, } \\
\text { Ramos- } \\
\text { Valencia, \& } \\
\text { Ortiz [31] }\end{array}$ & 2008 & Puerto Rico & $\begin{array}{l}\text { Quantitative, cross } \\
\text { sectional }\end{array}$ & $\begin{array}{l}\text { To investigate } \\
\text { the association } \\
\text { between CS } \\
\text { and } \\
\text { breastfeeding } \\
\text { initiation }\end{array}$ & $\begin{array}{l}C S=598 \\
V D=1097\end{array}$ & $\begin{array}{l}\text { - CS was a barrier } \\
\text { to breastfeeding } \\
\text { initiation of } \\
\text { reproductive age } \\
\text { women in Puerto } \\
\text { Rico }\end{array}$ & $\begin{array}{l}\text { breastfeeding } \\
\text { initiation: } \\
\text { educational } \\
\text { attainment, } \\
\text { marital status, } \\
\text { and } \\
\text { employment } \\
\text { status. }\end{array}$ & $\begin{array}{l}\text { Moderate } \\
\text { quality }\end{array}$ \\
\hline 5 & $\begin{array}{l}\text { Chalmers } \\
\text { et al. [20] }\end{array}$ & 2010 & Canada & $\begin{array}{l}\text { Quantitative, cross } \\
\text { sectional }\end{array}$ & $\begin{array}{l}\text { To know the } \\
\text { correlation } \\
\text { between CS } \\
\text { and } \\
\text { breastfeeding }\end{array}$ & $\begin{array}{l}C S=2246 \\
V D=6296\end{array}$ & $\begin{array}{l}\text { - CS had less } \\
\text { mother-infant } \\
\text { contact } \\
\text { experiences. } \\
\text { - CS had less } \\
\text { mothers } \\
\text { breastfeeding } \\
\text { their babies at } \\
\text { almost all time } \\
\text { periods }\end{array}$ & & $\begin{array}{l}\text { Moderate } \\
\text { quality }\end{array}$ \\
\hline
\end{tabular}




\begin{tabular}{|c|c|c|c|c|c|c|c|c|c|}
\hline NO & Authors & Year & Country & Study Design & Objectives & Population & $\begin{array}{l}\text { Summary of } \\
\text { Results }\end{array}$ & $\begin{array}{l}\text { Other factors } \\
\text { associated } \\
\text { with breast } \\
\text { feeding } \\
\text { initiation and } \\
\text { exclusive } \\
\text { breastfeeding }\end{array}$ & $\begin{array}{l}\text { Summary } \\
\text { of Critical } \\
\text { Appraisal }\end{array}$ \\
\hline 6 & $\begin{array}{l}\text { Al-Sahab et } \\
\text { al. [21] }\end{array}$ & 2010 & Canada & $\begin{array}{l}\text { Quantitative, } \\
\text { cohort study }\end{array}$ & $\begin{array}{l}\text { To investigate } \\
\text { the rate of } \\
\text { breastfeeding } 6 \\
\text { months after } \\
\text { delivery }\end{array}$ & $\begin{array}{l}C S=1456 \\
V D=4146\end{array}$ & $\begin{array}{l}\text { - Exclusive } \\
\text { breastfeeding was } \\
\text { correlated with the } \\
\text { type of delivery. } \\
\text { - Pain and } \\
\text { discomfort due to } \\
\text { C-section effect } \\
\text { may prevent the } \\
\text { mother to } \\
\text { breastfed. }\end{array}$ & $\begin{array}{l}\text { Exclusive } \\
\text { breastfeeding } \\
6 \text { months } \\
\text { after delivery: } \\
\text { Mother's } \\
\text { education, } \\
\text { marital status, } \\
\text { smoking } \\
\text { during } \\
\text { pregnancy, } \\
\text { type of setting } \\
\text { of baby's } \\
\text { birth, Baby's } \\
\text { admission to } \\
\text { NICU, } \\
\text { employment } \\
\text { status. }\end{array}$ & $\begin{array}{l}\text { Moderate } \\
\text { quality }\end{array}$ \\
\hline 7 & $\begin{array}{l}\text { Zanardo et } \\
\text { al. [26] }\end{array}$ & 2010 & Italy & $\begin{array}{l}\text { Quantitative, } \\
\text { cohort study }\end{array}$ & $\begin{array}{l}\text { To evaluate } \\
\text { breastfeeding } \\
\text { rate } \\
\text { postdelivery up } \\
\text { to six months } \\
\text { after CS and } \\
\text { VD }\end{array}$ & $\begin{array}{l}C S=398 \\
V D=1496\end{array}$ & $\begin{array}{l}\text { - Both elective and } \\
\text { emergency CS } \\
\text { had negatively } \\
\text { effects on } \\
\text { breastfeeding. }\end{array}$ & & $\begin{array}{l}\text { Moderate } \\
\text { quality }\end{array}$ \\
\hline 8 & $\begin{array}{l}\text { Ahluwalia, } \\
\text { Li, \& } \\
\text { Morrow } \\
\text { [36] }\end{array}$ & 2012 & $\begin{array}{l}\text { United } \\
\text { States of } \\
\text { America }\end{array}$ & $\begin{array}{l}\text { Quantitative, } \\
\text { cohort study }\end{array}$ & $\begin{array}{l}\text { To know the } \\
\text { corelation } \\
\text { between type } \\
\text { of delivery and } \\
\text { breastfeeding }\end{array}$ & $\begin{array}{l}C S=489 \\
V D=1157\end{array}$ & $\begin{array}{l}\text { - No significant } \\
\text { correlation } \\
\text { between type of } \\
\text { delivery and } \\
\text { breastfeeding } \\
\text { initiation. } \\
\text { - Women with } \\
\text { assisted deliveries } \\
\text { need additional } \\
\text { support. }\end{array}$ & & $\begin{array}{l}\text { Moderate } \\
\text { quality }\end{array}$ \\
\hline 9 & $\begin{array}{l}\text { Liu, Zhang, } \\
\text { Liu, Li, \& Li } \\
\text { [23] }\end{array}$ & 2012 & China & $\begin{array}{l}\text { Quantitative, } \\
\text { cohort study }\end{array}$ & $\begin{array}{l}\text { To examine the } \\
\text { corelation } \\
\text { between type } \\
\text { of delivery and } \\
\text { method of } \\
\text { breastfeeding }\end{array}$ & $\begin{array}{l}C S= \\
22462 \\
V D= \\
409242\end{array}$ & $\begin{array}{l}\text { - Women who had } \\
\text { CS were less likely } \\
\text { to exclusively } \\
\text { breastfeed than } \\
\text { women who had } \\
\text { VD. }\end{array}$ & & $\begin{array}{l}\text { High } \\
\text { quality }\end{array}$ \\
\hline 10 & $\begin{array}{l}\text { Watt et al. } \\
\text { [22] }\end{array}$ & 2012 & Canada & $\begin{array}{l}\text { Quantitative, } \\
\text { cohort study }\end{array}$ & $\begin{array}{l}\text { To investigate } \\
\text { the relationship } \\
\text { between type } \\
\text { of delivery and } \\
\text { breastfeeding } \\
\text { initiation and } \\
\text { continuance up } \\
\text { to six weeks } \\
\text { after delivery }\end{array}$ & $\begin{array}{l}C S=826 \\
V D=1668\end{array}$ & $\begin{array}{l}\text { - The type of } \\
\text { delivery was not a } \\
\text { determining factor } \\
\text { of breastfeeding } \\
\text { initiation in the } \\
\text { early post- } \\
\text { discharge period. }\end{array}$ & & $\begin{array}{l}\text { Moderate } \\
\text { quality }\end{array}$ \\
\hline 11 & $\begin{array}{l}\text { Albokhary } \\
\& \text { James } \\
\text { [33] }\end{array}$ & 2014 & $\begin{array}{l}\text { Saudi } \\
\text { Arabia }\end{array}$ & $\begin{array}{l}\text { Quantitative, } \\
\text { Cross sectional }\end{array}$ & $\begin{array}{l}\text { To examine } \\
\text { whether the } \\
\text { type of delivery } \\
\text { influenced the } \\
\text { breastfeeding } \\
\text { practice }\end{array}$ & $\begin{array}{l}C S=30 \\
V D=30\end{array}$ & $\begin{array}{l}\text { - Women who had } \\
\text { CS were less likely } \\
\text { to initiate } \\
\text { breastfeeding and } \\
\text { likely to introduce } \\
\text { formula milk. } \\
\text { - Pain after birth } \\
\text { due to CS had } \\
\text { negative effects } \\
\text { on breastfeeding. }\end{array}$ & & $\begin{array}{l}\text { Moderate } \\
\text { quality }\end{array}$ \\
\hline
\end{tabular}




\begin{tabular}{|c|c|c|c|c|c|c|c|c|c|}
\hline NO & Authors & Year & Country & Study Design & Objectives & Population & $\begin{array}{l}\text { Summary of } \\
\text { Results }\end{array}$ & $\begin{array}{l}\text { Other factors } \\
\text { associated } \\
\text { with breast } \\
\text { feeding } \\
\text { initiation and } \\
\text { exclusive } \\
\text { breastfeeding }\end{array}$ & $\begin{array}{l}\text { Summary } \\
\text { of Critical } \\
\text { Appraisal }\end{array}$ \\
\hline 12 & $\begin{array}{l}\text { Berde \& } \\
\text { Yalcin [15] }\end{array}$ & 2016 & Nigeria & $\begin{array}{l}\text { Quantitative, } \\
\text { Cross sectional }\end{array}$ & $\begin{array}{l}\text { To recognize } \\
\text { the factors } \\
\text { associated } \\
\text { with Early } \\
\text { Initiation of } \\
\text { Breast Feeding } \\
\text { (EIBF) }\end{array}$ & $\begin{array}{l}C S=263 \\
V D= \\
11508\end{array}$ & $\begin{array}{l}\text { - Mothers who } \\
\text { had VD had three } \\
\text { times higher early } \\
\text { initiation of } \\
\text { breastfeeding } \\
\text { than mothers who } \\
\text { had CS. }\end{array}$ & $\begin{array}{l}\text { breastfeeding } \\
\text { initiation: } \\
\text { mother's age, } \\
\text { education, } \\
\text { ANC visit, } \\
\text { place of } \\
\text { delivery, baby } \\
\text { birth weight, } \\
\text { occupation, } \\
\text { wealth Index, } \\
\text { and type of } \\
\text { place of } \\
\text { residence }\end{array}$ & $\begin{array}{l}\text { High } \\
\text { quality }\end{array}$ \\
\hline 13 & $\begin{array}{l}\text { Hobbs, } \\
\text { Mannion, } \\
\text { McDonald, } \\
\text { Brockway, } \\
\text { \& Tough } \\
{[11]}\end{array}$ & 2016 & Canada & Cohort study & $\begin{array}{l}\text { To evaluate the } \\
\text { corelation } \\
\text { between type } \\
\text { of delivery and } \\
\text { breastfeeding } \\
\text { initiation }\end{array}$ & $\begin{array}{l}C S=739 \\
V D=2279\end{array}$ & $\begin{array}{l}\text { - There was no } \\
\text { significant } \\
\text { difference in the } \\
\text { breastfeeding } \\
\text { practice between } \\
\text { women who had } \\
\text { CS and women } \\
\text { who had VD. } \\
\text { - Women who had } \\
\text { CS were more } \\
\text { likely to } \\
\text { discontinue } \\
\text { breastfeeding } \\
\text { before three } \\
\text { months after } \\
\text { delivery. }\end{array}$ & & $\begin{array}{l}\text { High } \\
\text { quality }\end{array}$ \\
\hline 14 & $\begin{array}{l}\text { Kiani et al. } \\
{[16]}\end{array}$ & 2017 & Nicaragua & $\begin{array}{l}\text { Quantitative, cross } \\
\text { sectional }\end{array}$ & $\begin{array}{l}\text { To investigate } \\
\text { the corelation } \\
\text { between type } \\
\text { of delivery and } \\
\text { breastfeeding }\end{array}$ & $\begin{array}{l}C S=10 \\
V D=147\end{array}$ & $\begin{array}{l}\text { - There was no } \\
\text { difference in } \\
\text { breastfeeding } \\
\text { initiation between } \\
\text { mothers who had } \\
\text { CS and mothers } \\
\text { who had VD. } \\
\text { - Providing } \\
\text { prelacteal feeds } \\
\text { before early } \\
\text { initiation of } \\
\text { breastfeeding has } \\
\text { a negative effect } \\
\text { on breastfeeding. }\end{array}$ & $\begin{array}{l}\text { Breastfeeding } \\
\text { initiation: } \\
\text { mother's BMI } \\
\text { and mother's } \\
\text { age } \\
\text { Exclusive } \\
\text { breastfeeding } \\
6 \text { months } \\
\text { after delivery: } \\
\text { travel time to } \\
\text { health centre, } \\
\text { and weight of } \\
\text { baby at birth }\end{array}$ & $\begin{array}{l}\text { High } \\
\text { quality }\end{array}$ \\
\hline 15 & $\begin{array}{l}\text { Chen et al. } \\
\text { [5] }\end{array}$ & 2018 & China & $\begin{array}{l}\text { Quantitative, } \\
\text { cohort study }\end{array}$ & $\begin{array}{l}\text { To identify the } \\
\text { potential } \\
\text { effects of CS } \\
\text { on } \\
\text { breastfeeding } \\
\text { practices and } \\
\text { breastfeeding } \\
\text { duration }\end{array}$ & $\begin{array}{l}C S=387 \\
V D=567\end{array}$ & $\begin{array}{l}\text { - Mothers who } \\
\text { had CS reported a } \\
\text { low percentage of } \\
\text { exclusive } \\
\text { breastfeeding and } \\
\text { any breastfeeding } \\
\text { compared with } \\
\text { mothers who had } \\
\text { VD. } \\
\text { - CS also } \\
\text { shortened the } \\
\text { breastfeeding } \\
\text { duration. } \\
\text { - CS was also } \\
\text { associated with } \\
\text { delayed } \\
\text { breastfeeding } \\
\text { initiation and } \\
\text { giving formula } \\
\text { milk. }\end{array}$ & & $\begin{array}{l}\text { High } \\
\text { quality }\end{array}$ \\
\hline
\end{tabular}




\begin{tabular}{|c|c|c|c|c|c|c|c|c|c|}
\hline No & Authors & Year & Country & Study Design & Objectives & Population & $\begin{array}{l}\text { Summary of } \\
\text { Results }\end{array}$ & $\begin{array}{l}\text { Other factors } \\
\text { associated } \\
\text { with breast } \\
\text { feeding } \\
\text { initiation and } \\
\text { exclusive } \\
\text { breastfeeding }\end{array}$ & $\begin{array}{l}\text { Summary } \\
\text { of Critical } \\
\text { Appraisal }\end{array}$ \\
\hline 16 & $\begin{array}{l}\text { Ezeh et al. } \\
\text { [17] }\end{array}$ & 2019 & $\begin{array}{l}\text { Benin, } \\
\text { Burkina } \\
\text { Faso, Cabo } \\
\text { Verde, Cote } \\
\text { d'Ivoire, The } \\
\text { Gambia, } \\
\text { Ghana, } \\
\text { Guinea, } \\
\text { Guinea- } \\
\text { Bissau, } \\
\text { Liberia, } \\
\text { Mali, Niger, } \\
\text { Nigeria, } \\
\text { Senegal, } \\
\text { Sierra } \\
\text { Leone, and } \\
\text { Togo }\end{array}$ & $\begin{array}{l}\text { Quantitative, cross } \\
\text { sectional }\end{array}$ & $\begin{array}{l}\text { To investigate } \\
\text { the possible } \\
\text { characteristics } \\
\text { influencing } \\
\text { early initiation } \\
\text { of } \\
\text { breastfeeding } \\
\text { in } 13 \text { members } \\
\text { of ECOWAS }\end{array}$ & $\begin{array}{l}C S=2966 \\
V D= \\
73763\end{array}$ & $\begin{array}{l}\text { - Mothers who } \\
\text { received CS had } \\
\text { less early } \\
\text { initiation of } \\
\text { breastfeeding } \\
\text { than mothers who } \\
\text { received VD. }\end{array}$ & $\begin{array}{l}\text { Breastfeeding } \\
\text { initiation: } \\
\text { household } \\
\text { level, } \\
\text { mothers' first } \\
\text { delivery, } \\
\text { delivery } \\
\text { setting. }\end{array}$ & $\begin{array}{l}\text { High } \\
\text { quality }\end{array}$ \\
\hline 17 & $\begin{array}{l}\text { Zhang et } \\
\text { al.[7] }\end{array}$ & 2019 & China & $\begin{array}{l}\text { Quantitative, } \\
\text { cohort study }\end{array}$ & $\begin{array}{l}\text { To investigate } \\
\text { the correlation } \\
\text { of early } \\
\text { initiation of } \\
\text { breastfeeding } \\
\text { with long-term } \\
\text { breastfeeding } \\
\text { outcome after } \\
\text { CS }\end{array}$ & $\begin{array}{l}C S=333 \\
V D=315\end{array}$ & $\begin{array}{l}\text { - CS had a } \\
\text { negative effect on } \\
\text { early } \\
\text { breastfeeding } \\
\text { behaviors and } \\
\text { continued to have } \\
\text { long-term effects } \\
\text { on breastfeeding } \\
\text { outcomes. } \\
\text { - CS is not a } \\
\text { negative factor; } \\
\text { however, infants } \\
\text { who have feeding } \\
\text { difficulties at the } \\
\text { early stage of } \\
\text { breastfeeding will } \\
\text { have long-term } \\
\text { problems. }\end{array}$ & & $\begin{array}{l}\text { High } \\
\text { quality }\end{array}$ \\
\hline 18 & $\begin{array}{l}\text { Ragusa et } \\
\text { al.[12] }\end{array}$ & 2020 & Italy & $\begin{array}{l}\text { Quantitative, cross } \\
\text { sectional }\end{array}$ & $\begin{array}{l}\text { To assess the } \\
\text { percentage of } \\
\text { breastfeeding } \\
\text { during hospital } \\
\text { stay }\end{array}$ & $\begin{array}{l}C S=1488 \\
V D=2325\end{array}$ & $\begin{array}{l}\text { - Exclusive } \\
\text { breastfeeding was } \\
\text { less adopted in } \\
\text { women who had } \\
\text { CS than in women } \\
\text { who had VD. }\end{array}$ & & $\begin{array}{l}\text { Moderate } \\
\text { quality }\end{array}$ \\
\hline 19 & Ali et al.[28] & 2020 & Bangladesh & $\begin{array}{l}\text { Quantitative, cross } \\
\text { sectional }\end{array}$ & $\begin{array}{l}\text { To evaluate the } \\
\text { relationship of } \\
\text { childbirth } \\
\text { location and } \\
\text { type of delivery } \\
\text { with early } \\
\text { breastfeeding } \\
\text { practices }\end{array}$ & $\begin{array}{l}C S=359 \\
V D=2362\end{array}$ & $\begin{array}{l}\text { - Early initiation of } \\
\text { breastfeeding was } \\
\text { less frequent in } \\
\text { women who had } \\
\text { CS than in women } \\
\text { who had VD. }\end{array}$ & $\begin{array}{l}\text { Initiation of } \\
\text { breastfeeding: } \\
\text { place of } \\
\text { delivery. }\end{array}$ & $\begin{array}{l}\text { High } \\
\text { quality }\end{array}$ \\
\hline 20 & $\begin{array}{l}\text { Apanga \& } \\
\text { Kumbeni } \\
{[18]}\end{array}$ & 2020 & Ghana & $\begin{array}{l}\text { Quantitative, cross } \\
\text { sectional }\end{array}$ & $\begin{array}{l}\text { To examine the } \\
\text { percentage of } \\
\text { breastfeeding } \\
\text { and factors } \\
\text { related to the } \\
\text { initiation of } \\
\text { breastfeeding }\end{array}$ & $\begin{array}{l}C S=1663 \\
V D= \\
13642\end{array}$ & $\begin{array}{l}\text { - Breastfeeding } \\
\text { initiation was less } \\
\text { in women who } \\
\text { had CS than in } \\
\text { women who had } \\
\text { VD. }\end{array}$ & $\begin{array}{l}\text { Initiation of } \\
\text { breastfeeding: } \\
\text { delivery } \\
\text { assisted by } \\
\text { skilled } \\
\text { attendant, } \\
\text { Planned } \\
\text { pregnancy, } \\
\text { and weight of } \\
\text { baby at birth }\end{array}$ & $\begin{array}{l}\text { High } \\
\text { quality }\end{array}$ \\
\hline 21 & $\begin{array}{l}\text { Chehab, } \\
\text { Nasreddine, } \\
\text { Zgheib, \& } \\
\text { Forman } \\
\text { [30] }\end{array}$ & 2020 & Lebanon & $\begin{array}{l}\text { Quantitative, cross } \\
\text { sectional }\end{array}$ & $\begin{array}{l}\text { To assess the } \\
\text { frequency of } \\
\text { and factors } \\
\text { related to } \\
\text { exclusive } \\
\text { breastfeeding } \\
\text { at } 40 \text { days and } \\
\text { at six months }\end{array}$ & $\begin{array}{l}C S=424 \\
V D=517\end{array}$ & $\begin{array}{l}\text { - The frequency of } \\
\text { exclusive } \\
\text { breastfeeding was } \\
\text { higher in the VD } \\
\text { group than in the } \\
\text { CS group at } 40 \\
\text { days and at six } \\
\text { months }\end{array}$ & $\begin{array}{l}\text { exclusive } \\
\text { breastfeeding } \\
\text { at } 40 \text { days } \\
\text { and at six } \\
\text { months: } \\
\text { family } \\
\text { monthly } \\
\text { income and } \\
\text { educational } \\
\text { level. }\end{array}$ & $\begin{array}{l}\text { High } \\
\text { quality }\end{array}$ \\
\hline
\end{tabular}




\begin{tabular}{|c|c|c|c|c|c|c|c|c|c|}
\hline No & Authors & Year & Country & Study Design & Objectives & Population & $\begin{array}{l}\text { Summary of } \\
\text { Results }\end{array}$ & $\begin{array}{l}\text { Other factors } \\
\text { associated } \\
\text { with breast } \\
\text { feeding } \\
\text { initiation and } \\
\text { exclusive } \\
\text { breastfeeding }\end{array}$ & $\begin{array}{l}\text { Summary } \\
\text { of Critical } \\
\text { Appraisal }\end{array}$ \\
\hline 22 & $\begin{array}{l}\text { Gedefaw, } \\
\text { Goedert, } \\
\text { Abebe, \& } \\
\text { Demis [19] }\end{array}$ & 2020 & Ethiopia & $\begin{array}{l}\text { Quantitative, cross } \\
\text { sectional }\end{array}$ & $\begin{array}{l}\text { To assess the } \\
\text { impact of CS } \\
\text { on } \\
\text { breastfeeding } \\
\text { initiation }\end{array}$ & $\begin{array}{l}C S=165 \\
V D=6950\end{array}$ & $\begin{array}{l}\text { - CS was a } \\
\text { significant factor } \\
\text { associated with } \\
\text { the late initiation } \\
\text { of breastfeeding }\end{array}$ & & $\begin{array}{l}\text { Moderate } \\
\text { quality }\end{array}$ \\
\hline 23 & $\begin{array}{l}\text { Hoang } \\
\text { Nguyen et } \\
\text { al.[37] }\end{array}$ & 2020 & Vietnam & $\begin{array}{l}\text { Quantitative, } \\
\text { cohort study }\end{array}$ & $\begin{array}{l}\text { To evaluate the } \\
\text { effects of CS } \\
\text { on } \\
\text { breastfeeding } \\
\text { practices from } \\
\text { delivery to } 12 \\
\text { months } \\
\text { postpartum }\end{array}$ & $\begin{array}{l}C S=654 \\
V D=1061\end{array}$ & $\begin{array}{l}\text { - There were lower } \\
\text { rates of early } \\
\text { initiation of } \\
\text { breastfeeding by } \\
\text { mothers who } \\
\text { underwent CS due } \\
\text { to prelacteal } \\
\text { feeding of their } \\
\text { infants. }\end{array}$ & & $\begin{array}{l}\text { Moderate } \\
\text { quality }\end{array}$ \\
\hline 24 & $\begin{array}{l}\text { Paksoy } \\
\text { Erbaydar \& } \\
\text { Erbaydar } \\
\text { [35] }\end{array}$ & 2020 & Turkey & $\begin{array}{l}\text { Quantitative, } \\
\text { cohort study }\end{array}$ & $\begin{array}{l}\text { To determine } \\
\text { the relationship } \\
\text { between CS } \\
\text { and early } \\
\text { breastfeeding } \\
\text { practices } \\
\text { among } \\
\text { primiparas }\end{array}$ & $\begin{array}{l}C S=417 \\
V D=360\end{array}$ & $\begin{array}{l}\text { - Women who had } \\
\text { CS had late } \\
\text { breastfeeding } \\
\text { initiation and non- } \\
\text { exclusive } \\
\text { breastfeeding } \\
\text { during the three } \\
\text { days following } \\
\text { delivery. }\end{array}$ & & $\begin{array}{l}\text { Moderate } \\
\text { quality }\end{array}$ \\
\hline 25 & $\begin{array}{l}\text { Baxter, } \\
2006 \text { [24] }\end{array}$ & 2006 & England & $\begin{array}{l}\text { Qualitative, focus } \\
\text { group } \\
\text { discussion }\end{array}$ & $\begin{array}{l}\text { To explore the } \\
\text { experiences of } \\
\text { feeding CS } \\
\text { babies }\end{array}$ & $\mathrm{CS}=11$ & $\begin{array}{l}\text { - The reasons for } \\
\text { stopping } \\
\text { breastfeeding } \\
\text { were the } \\
\text { perception of } \\
\text { insufficient milk } \\
\text { and } \\
\text { inconvenience, } \\
\text { difficulty with } \\
\text { attachment, pain, } \\
\text { and lack of } \\
\text { support }\end{array}$ & & $\begin{array}{l}\text { Moderate } \\
\text { quality }\end{array}$ \\
\hline 26 & $\begin{array}{l}\text { Chaplin et } \\
\text { al. [27] }\end{array}$ & 2016 & $\begin{array}{l}\text { Queensland, } \\
\text { Australia }\end{array}$ & $\begin{array}{l}\text { Qualitative, } \\
\text { interpretive } \\
\text { phenomenological } \\
\text { research }\end{array}$ & $\begin{array}{l}\text { To explore the } \\
\text { experiences of } \\
\text { women with } \\
\text { breastfeeding } \\
\text { problems }\end{array}$ & $C S=8$ & $\begin{array}{l}\text { - The problems of } \\
\text { breastfeeding } \\
\text { practice after CS } \\
\text { were anesthetic } \\
\text { recovery, lack of } \\
\text { true skin-to-skin } \\
\text { contact, } \\
\text { separation of } \\
\text { mother and baby, } \\
\text { inconsistent } \\
\text { information, } \\
\text { inadequate } \\
\text { support, } \\
\text { unnecessary milk } \\
\text { formula } \\
\text { supplementation } \\
\text { and feelings of } \\
\text { failure }\end{array}$ & & $\begin{array}{l}\text { High } \\
\text { quality }\end{array}$ \\
\hline 27 & $\begin{array}{l}\text { Tully, } \\
\text { Kristin P. \& } \\
\text { Ball [25] }\end{array}$ & 2014 & $\begin{array}{l}\text { Northeast } \\
\text { England }\end{array}$ & $\begin{array}{l}\text { Qualitative, } \\
\text { semistructured, } \\
\text { open-ended } \\
\text { interview }\end{array}$ & $\begin{array}{l}\text { To examine the } \\
\text { maternal } \\
\text { perspectives of } \\
\text { mechanisms } \\
\text { that contribute } \\
\text { to the difficulty } \\
\text { of early } \\
\text { breastfeeding } \\
\text { after CS }\end{array}$ & $\mathrm{CS}=115$ & $\begin{array}{l}\text { - The barriers to } \\
\text { breastfeeding } \\
\text { after CS were } \\
\text { maternal mobility } \\
\text { limitations, } \\
\text { positioning } \\
\text { difficulties, and } \\
\text { frustration at the } \\
\text { need for } \\
\text { assistance. }\end{array}$ & & $\begin{array}{l}\text { High } \\
\text { quality }\end{array}$ \\
\hline
\end{tabular}

Figure 2 shows that the percentages of early initiation of breastfeeding ( $\leq 1 \mathrm{hr}$ ) were higher in the mothers who had VD than in the mothers who had CSection. Most of the studies showed that more than $50 \%$ the mothers who had VD had early initiation of breastfeeding and only two studies reported an initiation below $50 \%$.

As for the mothers who had C-Section, nine studies reported that the rate of early initiation of breastfeeding were below $50 \%$. In addition, Figure 2 shows a large difference in the percentages of early initiation of breastfeeding between VD and C-section, and only 1 article showed a small difference in the rate of 
early initiation of breastfeeding between these two groups [16].

Most of the studies reported that the rate of exclusive breastfeeding at hospital discharge were more than $50 \%$ in the mothers who had VD and more than $30 \%$ in the mothers who had C-Section (Figure 4). Only two studies from Vietnam and Taiwan showed that the rate of exclusive breastfeeding at hospital discharge were below $25 \%$ in both the mothers who had VD and the mothers who had C-section [34,37]. Two studies showed a large difference in the rate of early initiation of breastfeeding between the mothers who had VD and the mothers who had C-section [7,12].

Figure 8 shows that the percentages of any breastfeeding 6 months after delivery in most studies were more than $50 \%$ for both the VD and C-section groups. There were almost same percentage between vaginal delivery and C-section of any breastfeeding 6 months after delivery [5,30,37].

As for the qualitative study, we synthesized the findings from three articles on barriers to breastfeeding experience after C-section. The categories were mother's physical discomfort, low self-efficacy, lack of breastfeeding knowledge, and inadequate support from a healthcare provider. The subcategory of these three articles is shown in Table 2.

Table 3

Themes and quotes of breastfeeding experiences after C-Section

\begin{tabular}{|c|c|c|c|}
\hline Theme & Category & Subcategory & Quotes \\
\hline \multirow{13}{*}{$\begin{array}{l}\text { Barriers to } \\
\text { breastfeeding } \\
\text { after CS }\end{array}$} & $\begin{array}{l}\text { Mother's physical } \\
\text { discomfort }\end{array}$ & $\begin{array}{l}\text { Limited maternal } \\
\text { mobility }\end{array}$ & "I can't get up... felt bit let down by that [limited mobility]" [25] \\
\hline & & $\begin{array}{l}\text { Positioning } \\
\text { difficulties }\end{array}$ & "Very traumatised after labour, baby did not latch properly and I found it painful" [24] \\
\hline & & Pain of scare & "Too painful to lift and carry baby post CS" [24,27] \\
\hline & & & "I sort of moved to get up on \\
\hline & & & the bed or to sort of move down a bit to go to sleep then I got \\
\hline & & & the pain" [27] \\
\hline & Low self-efficacy & Feelings of failure & $\begin{array}{l}\text { "I don't want to try anymore... feel stressed and the baby has mucus. I'm disappointed } \\
\text { that I couldn't [breast feed]." [25] }\end{array}$ \\
\hline & $\begin{array}{l}\text { Lack of } \\
\text { breastfeeding } \\
\text { knowledge }\end{array}$ & False belief & $\begin{array}{l}\text { "Not having enough breast milk - baby was still hungry no matter how long I fed her" } \\
\text { [24] }\end{array}$ \\
\hline & & $\begin{array}{l}\text { Misperception of } \\
\text { insufficient milk }\end{array}$ & $\begin{array}{l}\text { "I was unsure that I was giving enough milk as she was not gaining substantial weight, } \\
\text { also when breastfeeding I could hear the wind in her stomach" [24] }\end{array}$ \\
\hline & Inadequate support & Separation of & "they just showed me the baby in theatre when he first \\
\hline & provider & 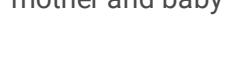 & $\begin{array}{l}\text { came out. I don't think I got to touch him.. . I thought I would have been allowed to } \\
\text { touch him" [27] }\end{array}$ \\
\hline & & $\begin{array}{l}\text { Unnecessary } \\
\text { formula } \\
\text { supplementation }\end{array}$ & $\begin{array}{l}\text { "after 24-48 hours I just couldn't get her to latch so I started expressing but then I } \\
\text { wasn't expressing much at all... so we had to go and get the formula" [27] }\end{array}$ \\
\hline & & $\begin{array}{l}\text { Lack of } \\
\text { professional skills } \\
\text { on breast latching }\end{array}$ & $\begin{array}{l}\text { "I received much conflicting advice from ward staff with each shift change and feel } \\
\text { this prevented me from adequately latching my baby and therefore being able to } \\
\text { breastfeed her" [24] }\end{array}$ \\
\hline
\end{tabular}

\section{Discussion}

\subsection{Low percentage of breastfeeding after C-Section}

This scoping review synthesized data of breastfeeding after C-Section from 1990 to 2020. Surprisingly, the results showed that the percentage of breastfeeding after $\mathrm{C}$-section did not improve compared with the results of a previous study by Prior et al in 2012 [6]. Prior et al. conducted a systematic review of studies on breastfeeding after C-section published from 1983 to 2011 from 33 countries. They reported that unsuccessful or delayed breastfeeding initiation was associated with C-section [6].

The present results showed a large difference in the percentage of early initiation of breastfeeding between the VD and C-section groups (Figure 2). Several factors have been reported to cause the delayed breastfeeding initiation after C-Section. These include the mother's physical discomfort after delivery, low self-efficacy, and lack of breastfeeding knowledge, as well as the inadequate support from healthcare providers [24,25,27]. Limited mobility and difficult mother-baby attachment owing to the pain after C-section impair the mother's ability to breastfeed her baby. Moreover, the absence of a rooming-in policy in a hospital/clinic after C-section and instead keeping the baby in a nursery room further delays the early initiation of breastfeeding [24, 26, 29,37]. Chaplin et al. also observed that most infants born by C-section were given formula milk in a nursery room before they were given to their mothers [27]. However, rooming-in is still raising a debate. Rooming-in can boost breastfeeding and self-efficacy [38,39]. On the other hand, rooming-in has been reported to

Page $9 / 18$ 
disturb well-rested mothers after delivery [40]. In their systematic review, Jaafar, Lee \& Ho showed that rooming-in was associated with successful breastfeeding initiation [41].

In Figure 4, two studies from Vietnam and Taiwan showed that the rate of exclusive breastfeeding at hospital discharge were below $25 \%$ in both the VD and C-Section groups [34,37]. This phenomenon in Taiwan is rooted in the traditional cultural belief of Chinese that pregnancy and delivery are considered as a tiring process which weakens the physical condition of women. Therefore, it is believed that women should take sufficient rest in bed after delivery. Moreover, mothers believe that there was insufficient milk for infants before breast engorgement [34]. In Vietnam, the low percentage of breastfeeding was due to the high rate of prelacteal feeding (particularly formula milk) and usage of antibiotics after C-Section. Mothers will delay breastfeeding to avoid passing on the antibiotics to their infants [37].

As for exclusive breastfeeding at six months after delivery, the results showed that the rate of exclusive breastfeeding were under $80 \%$. The reasons for the low percentages were returning to work, less milk production, and the introduction of solid food [21,24]. Healthcare providers should point out that the first period of hospitalization is very important to the successful continuance of breastfeeding

\subsection{Forms of support to increase breastfeeding after C-section}

Barriers to breastfeeding after $\mathrm{C}$-section can be overcome with appropriate assistance and breastfeeding education. For assistance, professional healthcare providers can provide physical and mental support to mothers in performing breastfeeding after C-section, particularly in the early postpartum period.

Skin-to-skin contact (SSC) is a form of support to increase successful breastfeeding after C-Section [42]. Healthcare providers can help administer SSC after C-Section to increase the mother's confidence and intimacy with her baby. Moran-Peter et al. explained that SSC contact after delivery can enhance exclusive breastfeeding practices [43].

As for the limited mobility and pain caused by surgery, the use of pain control (analgesia) has been recommended [44]. Healthcare providers can introduce different kinds of painkiller such suppository, oral medicine, and epidural anaesthesia. Tiredness was also reported to delay breastfeeding. Therefore, physical support from nurses or families is needed to help in the mother-baby attachment for breastfeeding and to build the confidence of mothers in breastfeeding their baby.

\subsection{Provision of evidence through breastfeeding education}

Providing counselling, motivation, and education to mothers about breastfeeding is an important task by healthcare providers. This is especially crucial in terms of providing adequate information about the breastmilk process, myths, and mother mobilization. Evidence suggests that breastfeeding education is effective in increasing both the rate of breastfeeding initiation and breastfeeding duration [45]. Lumbiganon et al. showed that breastfeeding education in antenatal care for mothers and spouses/families can have a marked effect on breastfeeding at the postpartum period [46].

Breastfeeding education is not only for mothers and their families, but also for healthcare providers. As science and research are constantly evolving, healthcare providers should receive continuing education, particularly on lactation knowledge and professional assistance on breastfeeding after Csection. A regular update on the knowledge of healthcare providers can be considered as part of a successful breastfeeding program.

Recently, healthcare providers have also introduced a decision-making aid on breastfeeding to help mothers decide whether to breastfeed their babies after delivery. In 2020, the Ottawa Hospital Research Institute launched a breastfeeding decision aid that can be used by healthcare providers. Breastfeeding education regarding the proper position and baby attachment has been given following the postnatal period. Home visits by peer counsellors have also been shown to significantly increase exclusive breastfeeding 12 and 24 weeks postpartum [47].

\subsection{Adopting a baby friendly hospital initiative program}

In 1991, the WHO and United Nations International Children's Emergency Fund promoted the early initiation of breastfeeding through the Baby Friendly Hospital Initiative (BFHI) programme. This programme introduced breastfeeding domains such as no infant formula, promotion and support (prenatal breastfeeding education), and mother-baby rooming-in throughout the hospital [48].

The policy of the BFHI programme can be adopted by maternity clinics and hospitals to improve the breastfeeding practices of mothers who gave birth by VD or C-section. Thus, it is crucial to support mothers to breastfeed just after delivery and avoid formula milk. Rooming-in is considered as one of the approaches to creating a bond between the mother and the baby, making it easier for mothers to breastfeed their baby on demand. However, there is still no definitive evidence regarding the corelation of rooming-in with breastfeeding duration [41]. Optimal breastfeeding care for mothers who had C-section may increase their rate of early initiation of breastfeeding.

\subsection{Strengths and Limitations}

This study has several limitations. It was limited to peer-reviewed studies published in English. Specifically, it included only studies reporting on the percentages of breastfeeding after C-section and VD. Moreover, only three qualitative studies were included. Despite these limitations, the strengths of this study were its rigorous methodological frameworks for conducting and reporting this scoping review and the meticulous review of studies for critical appraisal by three independent reviewers.

\section{Conclusion}


This scoping review found a low percentage of breastfeeding among mothers who had $\mathrm{C}$-section. The underlying reasons for the large differences in the percentages of breastfeeding after C-section and VD were the physical discomfort, low self-efficacy, and lack of breastfeeding knowledge of mothers, as well as the inadequate support from healthcare providers. Pain control, SSC, breastfeeding education in antenatal care, development of a breastfeeding decision aid, and implementing a baby friendly hospital policy are some important approaches to improving breastfeeding after C-section.

\section{Abbreviations}

CS/C-section

Cesarean section

PRISMA-ScR

Preferred Reporting Items for Systematic Reviews and Meta- Analyses extension for scoping reviews

VD

Vaginal delivery

WHO

World Health Organization

JBI

Joanna Briggs Institutes

SSC

Skin-to-skin contact

BFHI

Baby Friendly Hospital Initiative

\section{Declarations}

\section{Ethical approval}

Ethical approval was not required for this paper

\section{Consent for publication}

Not applicable.

\section{Competing interests}

The authors declare that they have no competing interest associated with this study.

\section{Funding}

Japan Society for the Promotion of Science (JSPS) Core-to-Core Program, Asia-Africa Science Platforms (2021-2024, PI: Shigeko Horiuchi).

\section{Author information}

Yunefit Ulfa, Ph.D.

Graduate School of Nursing Science, St. Luke's International University, Tokyo Japan

E-mail: 17dn901@slcn.ac.jp

Naoko Maruyama, RN, CNM, PhD

Graduate School of Nursing Science, St. Luke's International University, Tokyo Japan

E-mail: naoko-maruyama@slcn.ac.jp

Yumiko Igarashi, RN, CNM

Graduate School of Nursing Science, St. Luke's International University, Tokyo Japan

E-mail: 19dn002@slcn.ac.jp

Shigeko Horiuchi, RN, CNM, PhD

Graduate School of Nursing Science, St. Luke's International University, Tokyo Japan 
E-mail: shigeko-horiuchi@slcn.ac.jp

\section{Corresponding author}

Correspondence to Yunefit Ulfa

E-mail: 17dn901@slcn.ac.jp

\section{Author contributions}

Y.U. contributed to the conception, formal analysis, methodology, software, and writing of the original draft presentation and design of this study; N.M. was involved in the critical appraisal, writing-review and editing; Y.I. was involved in the critical appraisal, writing-review and editing; S.H. was involved in developing the overall concept of the study, funding acquisition, supervision, validation, and writing-review and editing. All authors have read and approved the final manuscript.

\section{ACKNOWLEDGEMENTS}

We thank Dr. Edward Barroga (https://orcid.org/0000-0002-8920-2607), Medical and Nursing Science Editor and Professor of Academic Writing at St. Luke's International University for his editorial review of the manuscript.

\section{References}

1. Althabe F, Belizán J. Caesarean section: the paradox. The Lancet. 2006;368(9546):1472-1473.

2. Chien P. Global rising rates of caesarean sections. BJOG: An International Journal of Obstetrics \& Gynaecology. 2021;128(5):781-782.

3. Jenabi E, Khazaei S, Bashirian S, Aghababaei S, Matinnia N. Reasons for elective cesarean section on maternal request: a systematic review. The Journal of Maternal-Fetal \& Neonatal Medicine. 2019;33(22):3867-3872.

4. Antoine C, Young B. Cesarean section one hundred years 1920-2020: the Good, the Bad and the Ugly. Journal of Perinatal Medicine. 2020;49(1):5-16.

5. Chen C, Yan Y, Gao X, Xiang S, He Q, Zeng G et al. Influences of Cesarean Delivery on Breastfeeding Practices and Duration: A Prospective Cohort Study. Journal of Human Lactation. 2018;34(3):526-534.

6. Prior E, Santhakumaran S, Gale C, Philipps L, Modi N, Hyde M. Breastfeeding after cesarean delivery: a systematic review and meta-analysis of world literature. The American Journal of Clinical Nutrition. 2012;95(5):1113-1135.

7. Zhang F, Cheng J, Yan S, Wu H, Bai T. Early Feeding Behaviors and Breastfeeding Outcomes After Cesarean Section. Breastfeeding Medicine. 2019;14(5):325-333.

8. World Health Organization. Exclusive breastfeeding for six months best for babies everywhere. 2011. From https://www.who.int/news/item/15-012011-exclusive-breastfeeding-for-six-months-best-for-babies-everywhere

9. World Health Organization. Tracking progress for breastfeeding policies and programmes. Switzerland. 2017 From https://www.who.int/nutrition/publications/infantfeeding/global-bf-scorecard-2017.pdf

10. John J, Mistry S, Kebede G, Manohar N, Arora A. Determinants of early initiation of breastfeeding in Ethiopia: a population-based study using the 2016 demographic and health survey data. BMC Pregnancy and Childbirth. 2019;19(1).

11. Hobbs A, Mannion C, McDonald S, Brockway M, Tough S. The impact of caesarean section on breastfeeding initiation, duration and difficulties in the first four months postpartum. BMC Pregnancy and Childbirth. 2016;16(1).

12. Ragusa R, Giorgianni G, Marranzano M, Cacciola S, La Rosa V, Giarratana A et al. Breastfeeding in Hospitals: Factors Influencing Maternal Choice in Italy. International Journal of Environmental Research and Public Health. 2020;17(10):3575.

13. Joanna Briggs Institutes. (2017). Checklist for systematic reviews and research syntheses. From https://joannabriggs.org/ebp/critical|_appraisal_tools

14. Ouzzani M, Hammady H, Fedorowicz Z, Elmagarmid A. Rayyan-a web and mobile app for systematic reviews. Systematic Reviews. 2016;5(1).

15. Berde A, Yalcin S. Determinants of early initiation of breastfeeding in Nigeria: a population-based study using the 2013 demograhic and health survey data. BMC Pregnancy and Childbirth. 2016;16(1).

16. Kiani S, Rich K, Herkert D, Safon C, Pérez-Escamilla R. Delivery mode and breastfeeding outcomes among new mothers in Nicaragua. Maternal \& Child Nutrition. 2017;14(1):e12474.

17. Ezeh O, Ogbo F, Stevens G, Tannous W, Uchechukwu O, Ghimire P et al. Factors Associated with the Early Initiation of Breastfeeding in Economic Community of West African States (ECOWAS). Nutrients. 2019;11(11):2765.

18. Apanga P, Kumbeni M. RETRACTED ARTICLE: Prevalence and predictors of timely initiation of breastfeeding in Ghana: an analysis of $2017-2018$ multiple indicator cluster survey. International Breastfeeding Journal. 2020;15(1).

19. Gedefaw G, Goedert M, Abebe E, Demis A. Effect of cesarean section on initiation of breast feeding: Findings from 2016 Ethiopian Demographic and Health Survey. PLOS ONE. 2020;15(12):e0244229.

20. Chalmers B, Kaczorowski J, Darling E, Heaman M, Fell D, O’Brien B et al. Cesarean and Vaginal Birth in Canadian Women: A Comparison of Experiences. Birth. 2010;37(1):44-49.

Page $12 / 18$ 
21. Al-Sahab B, Lanes A, Feldman M, Tamim H. Prevalence and predictors of 6-month exclusive breastfeeding among Canadian women: a national survey. BMC Pediatrics. 2010;10(1).

22. Watt S, Sword W, Sheehan D, Foster G, Thabane L, Krueger P et al. The Effect of Delivery Method on Breastfeeding Initiation from the The Ontario Mother and Infant Study (TOMIS) III. Journal of Obstetric, Gynecologic \& Neonatal Nursing. 2012;41(6):728-737.

23. Liu X, Zhang J, Liu Y, Li Y, Li Z. The Association between Cesarean Delivery on Maternal Request and Method of Newborn Feeding in China. PLoS ONE. 2012;7(5):e37336.

24. Baxter J. Women's experience of infant feeding following birth by caesarean section. British Journal of Midwifery. 2006;14(5):290-295.

25. Tully K, Ball H. Maternal accounts of their breast-feeding intent and early challenges after caesarean childbirth. Midwifery. 2014;30(6):712-719.

26. Zanardo V, Canella A, Maone R, Straface G. Bonding and breastfeeding after a cesarean delivery. Early Human Development. 2013;89:S56-S57.

27. Chaplin J, Kelly J, Kildea S. Maternal perceptions of breastfeeding difficulty after caesarean section with regional anaesthesia: A qualitative study. Women and Birth. 2016;29(2):144-152.

28. Ali N, Karim F, Billah S, Hoque D, Khan A, Hasan M et al. Are childbirth location and mode of delivery associated with favorable early breastfeeding practices in hard to reach areas of Bangladesh?. PLOS ONE. 2020;15(11):e0242135.

29. Vestermark V, Høgdall C, Birch M, Plenov G, Toftager-Larsen K. Influence of the mode of delivery on initiation of breast-feeding. European Journal of Obstetrics \& Gynecology and Reproductive Biology. 1991;38(1):33-38.

30. Chehab R, Nasreddine L, Zgheib R, Forman M. Exclusive breastfeeding during the 40-day rest period and at six months in Lebanon: a cross-sectional study. International Breastfeeding Journal. 2020;15(1).

31. Pérez-Ríos N, Ramos-Valencia G, Ortiz A. Cesarean Delivery as a Barrier for Breastfeeding Initiation: The Puerto Rican Experience. Journal of Human Lactation. 2008;24(3):293-302.

32. Wiklund I, Edman G, Andolf E. Cesarean section on maternal request: reasons for the request, self-estimated health, expectations, experience of birth and signs of depression among first-time mothers. Acta Obstetricia et Gynecologica Scandinavica. 2007;86(4):451-456.

33. Albokhary, A. A., \& James, J. P. Does cesarean section have an impact on the successful initiation of breastfeeding in Saudi Arabia? Saudi Medical Journal, 35(11), 1400-1403. (2014) Retrieved from https://www.embase.com/search/results? subaction=viewrecord\&id=L600453328\&from=export

34. Chien L, Tai C. Effect of Delivery Method and Timing of Breastfeeding Initiation on Breastfeeding Outcomes in Taiwan. Birth. 2007;34(2):123-130.

35. Paksoy Erbaydar N, Erbaydar T. Relationship between caesarean section and breastfeeding: evidence from the 2013 Turkey demographic and health survey. BMC Pregnancy and Childbirth. 2020;20(1).

36. Ahluwalia I, Li R, Morrow B. Breastfeeding Practices: Does Method of Delivery Matter?. Maternal and Child Health Journal. 2012;16(S2):231-237.

37. Hoang Nguyen P, Binns C, Vo Van Ha A, Nguyen C, Khac Chu T, Duong D et al. Caesarean delivery associated with adverse breastfeeding practices: a prospective cohort study. Journal of Obstetrics and Gynaecology. 2019;40(5):644-648.

38. Chiou S, Chen L, Yeh H, Wu S, Chien L. Early Skin-to-Skin Contact, Rooming-in, and Breastfeeding: A Comparison of the 2004 and 2011 National Surveys in Taiwan. Birth. 2014;41(1):33-38.

39. Jones R, Jones L, Feary A. The Effects of Single-Family Rooms on Parenting Behavior and Maternal Psychological Factors. Journal of Obstetric, Gynecologic \& Neonatal Nursing. 2016;45(3):359-370.

40. Keefe M. The Impact of Infant Rooming-In on Maternal Sleep at Night. Journal of Obstetric, Gynecologic \& Neonatal Nursing. 1988;17(2):122-126.

41. Jaafar S, Lee K, Ho J. Separate care for new mother and infant versus rooming-in for increasing the duration of breastfeeding. Cochrane Database of Systematic Reviews. 2012;.

42. Rowe-Murray H, Fisher J. Baby Friendly Hospital Practices: Cesarean Section is a Persistent Barrier to Early Initiation of Breastfeeding. Birth. 2002;29(2):124-131.

43. Moran-Peters J, Zauderer C, Goldman S, Baierlein J, Smith A. A Quality Improvement Project Focused on Women's Perceptions of Skin-to-Skin Contact After Cesarean Birth. Nursing for Women's Health. 2014;18(4):294-303.

44. Kintu A, Abdulla S, Lubikire A, Nabukenya M, Igaga E, Bulamba F et al. Postoperative pain after cesarean section: assessment and management in a tertiary hospital in a low-income country. BMC Health Services Research. 2019;19(1).

45. World Health Organization. Breastfeeding education for increased breastfeeding duration, 2013.. From https://www.who.int/elena/bbc/breastfeeding_education/en/

46. Lumbiganon P, Martis R, Laopaiboon M, Festin M, Ho J, Hakimi M. Antenatal breastfeeding education for increasing breastfeeding duration. Cochrane Database of Systematic Reviews. 2011;

47. Tylleskär T, Jackson D, Meda N, Engebretsen I, Chopra M, Diallo A et al. Exclusive breastfeeding promotion by peer counsellors in sub-Saharan Africa (PROMISE-EBF): a cluster-randomised trial. The Lancet. 2011;378(9789):420-427.

48. Pérez-Escamilla R. Breastfeeding in the 21st century: How we can make it work. Social science \& medicine 2020; $244,112331$. https://doi.org/10.1016/j.socscimed.2019.05.036

\section{Figures}




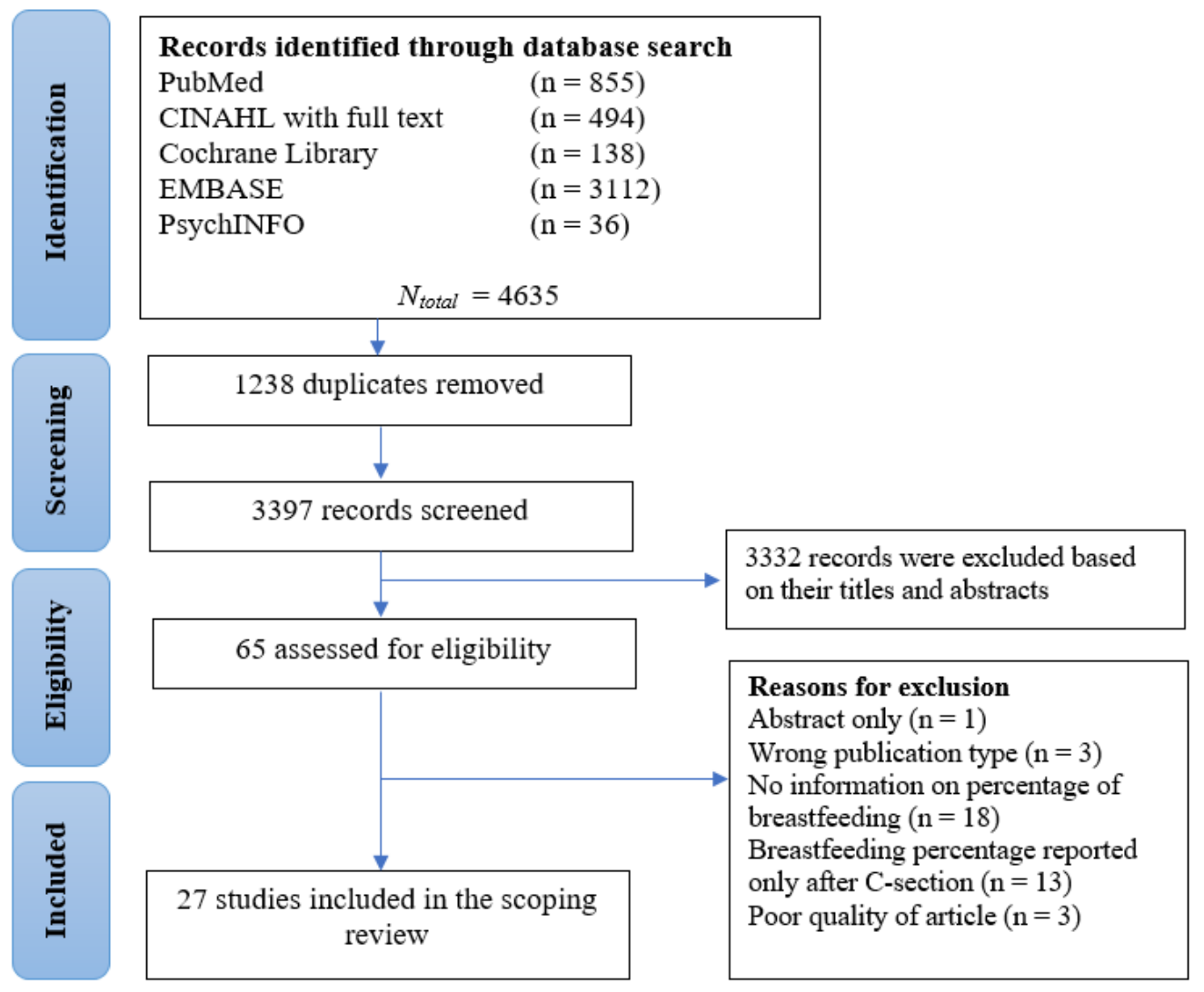

Figure 1

Flow diagram of study selection

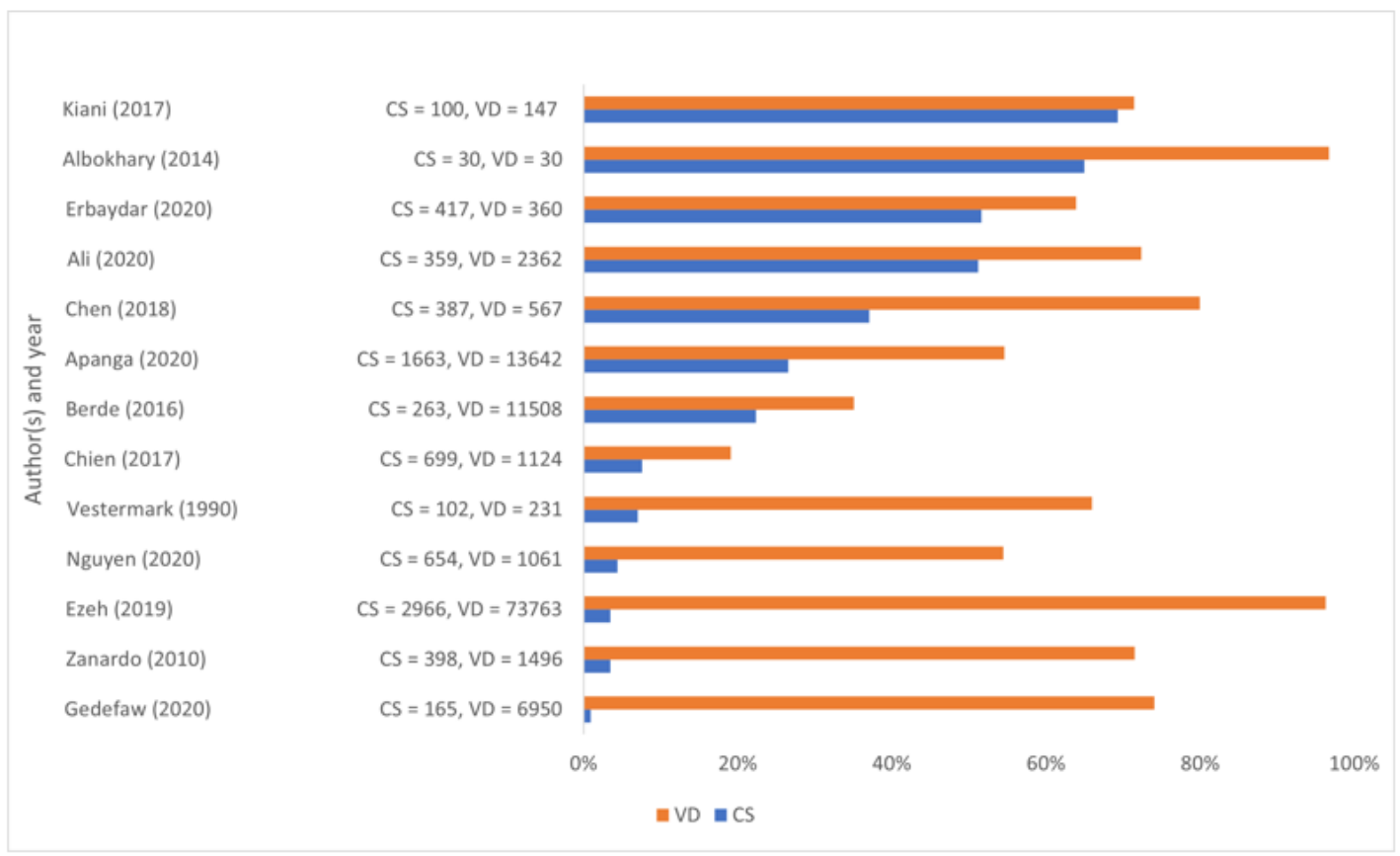

VD, Vaginal Delivery (population)

CS, C-Section (population)

Figure 2

Percentages of early initiation of breastfeeding ( $\leq 1 \mathrm{hr}$ ) 


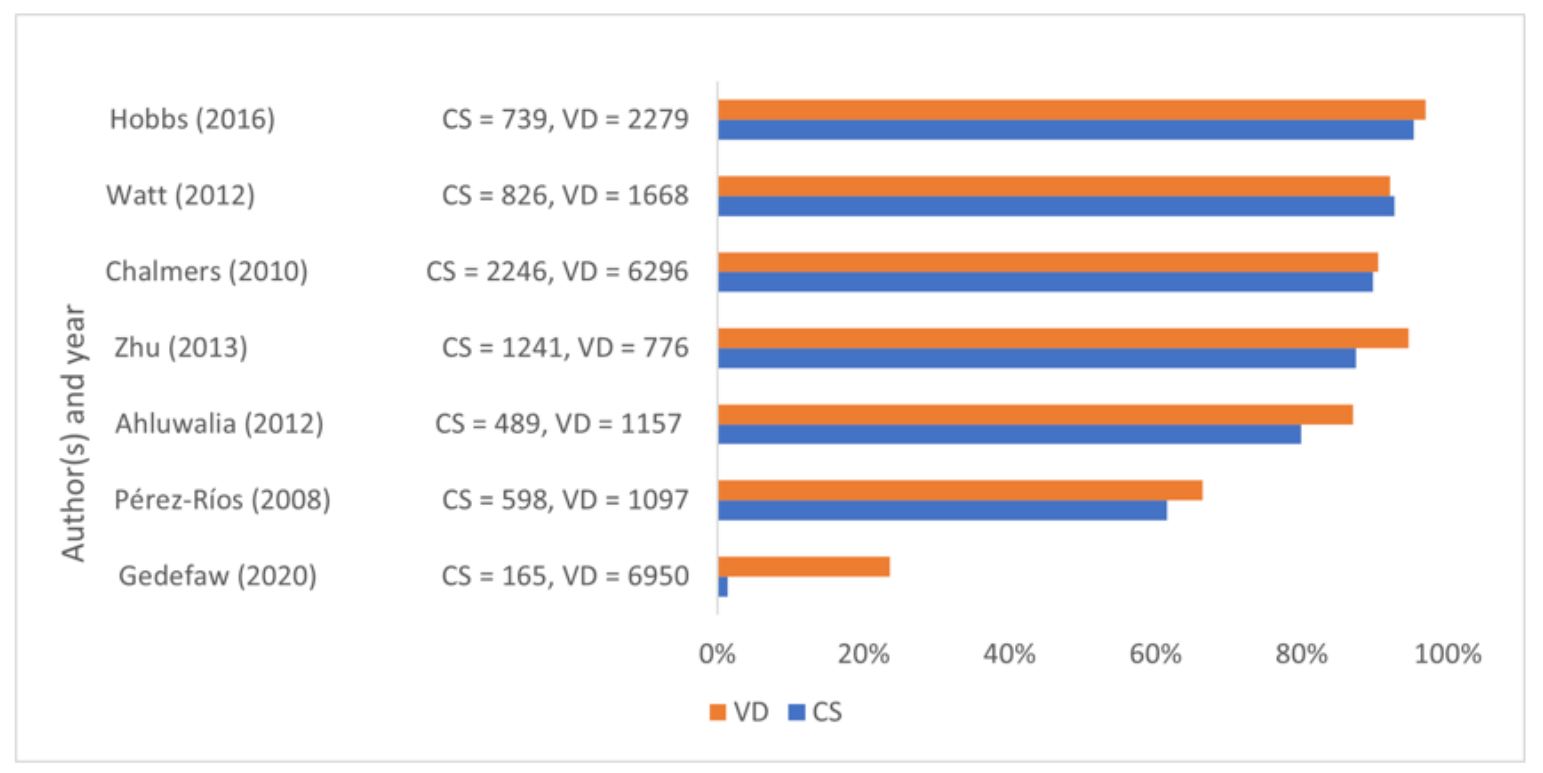

VD, Vaginal Delivery (population)

$\mathrm{CS}, \mathrm{C}$-Section (population)

\section{Figure 3}

Percentages of late initiation of breastfeeding ( $>1 \mathrm{hr}$ )

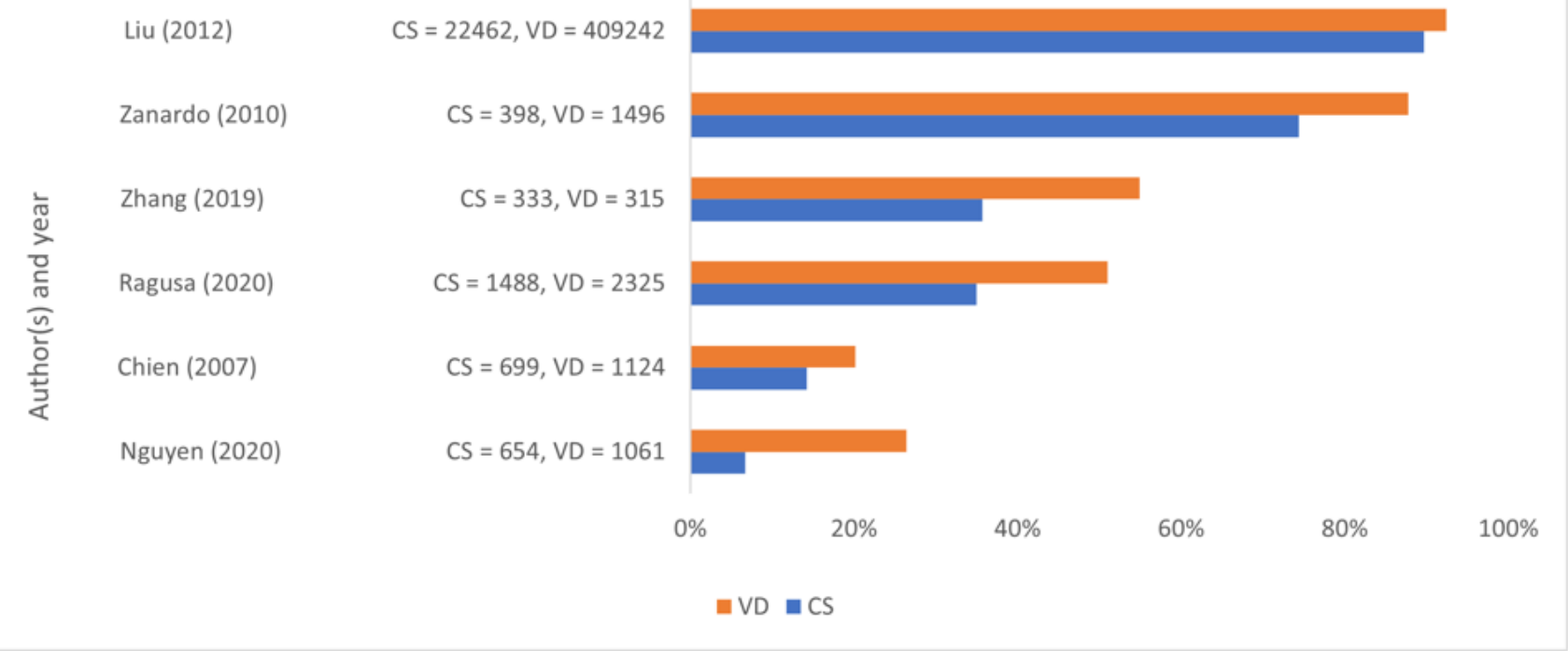

\section{VD, Vaginal Delivery (population)}

CS, C-Section (population)

\section{Figure 4}

Percentages of exclusive breastfeeding at hospital discharge 


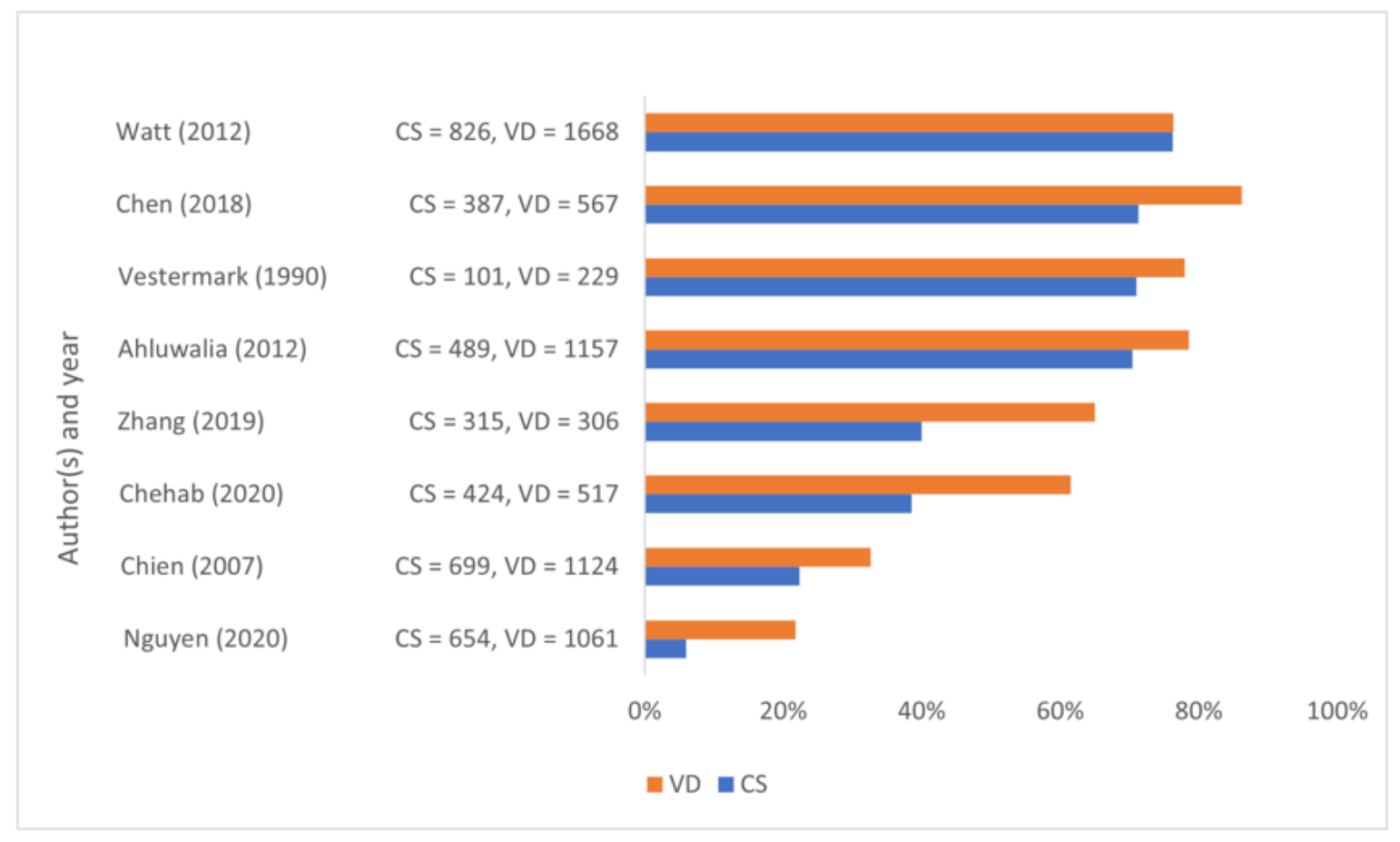

VD, Vaginal Delivery (population)

CS, C-Section (population)

\section{Figure 5}

Percentages of exclusive breastfeeding one month after delivery

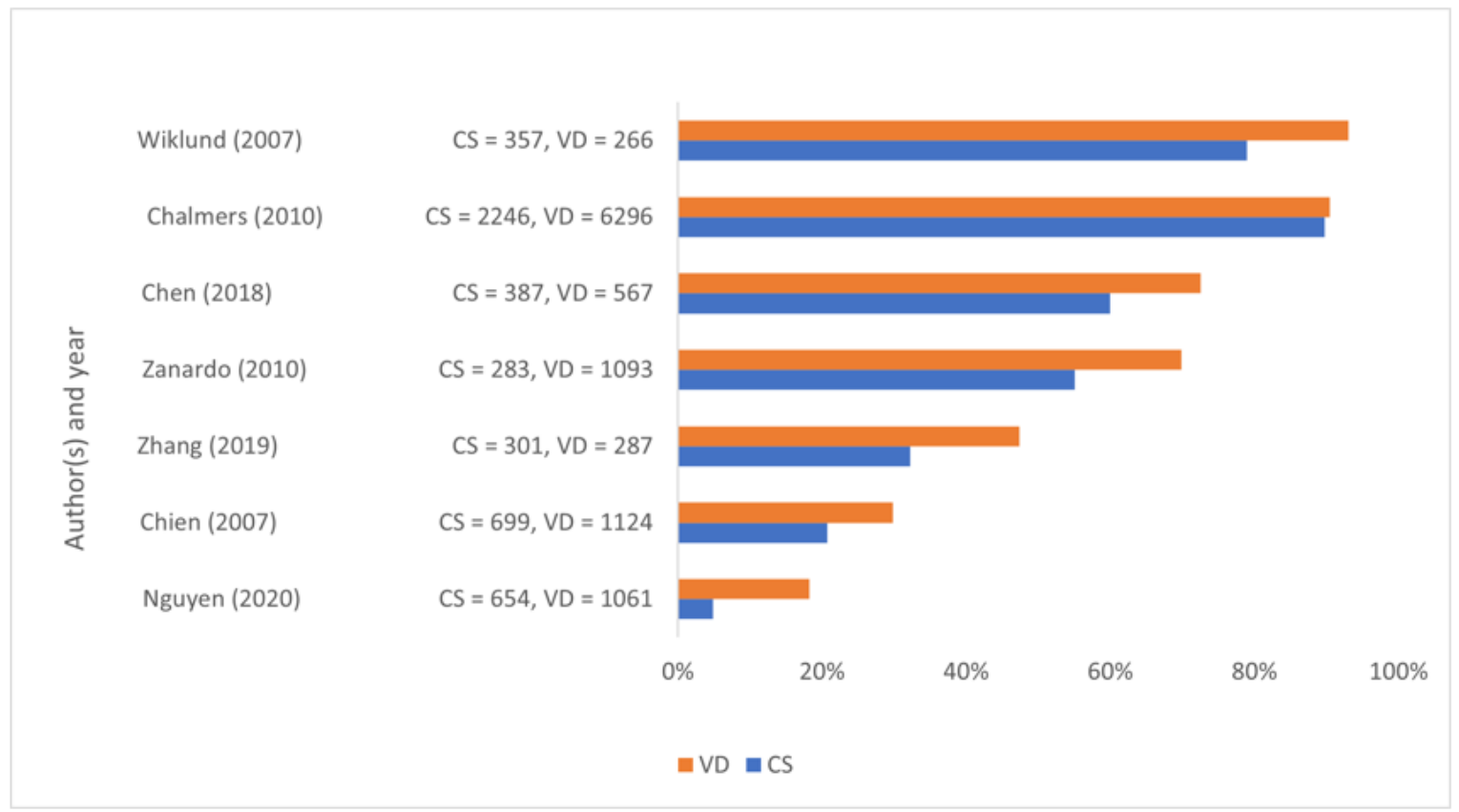

VD, Vaginal Delivery (population)

CS, C-Section (population)

\section{Figure 6}

Percentages of exclusive breastfeeding three months after delivery 


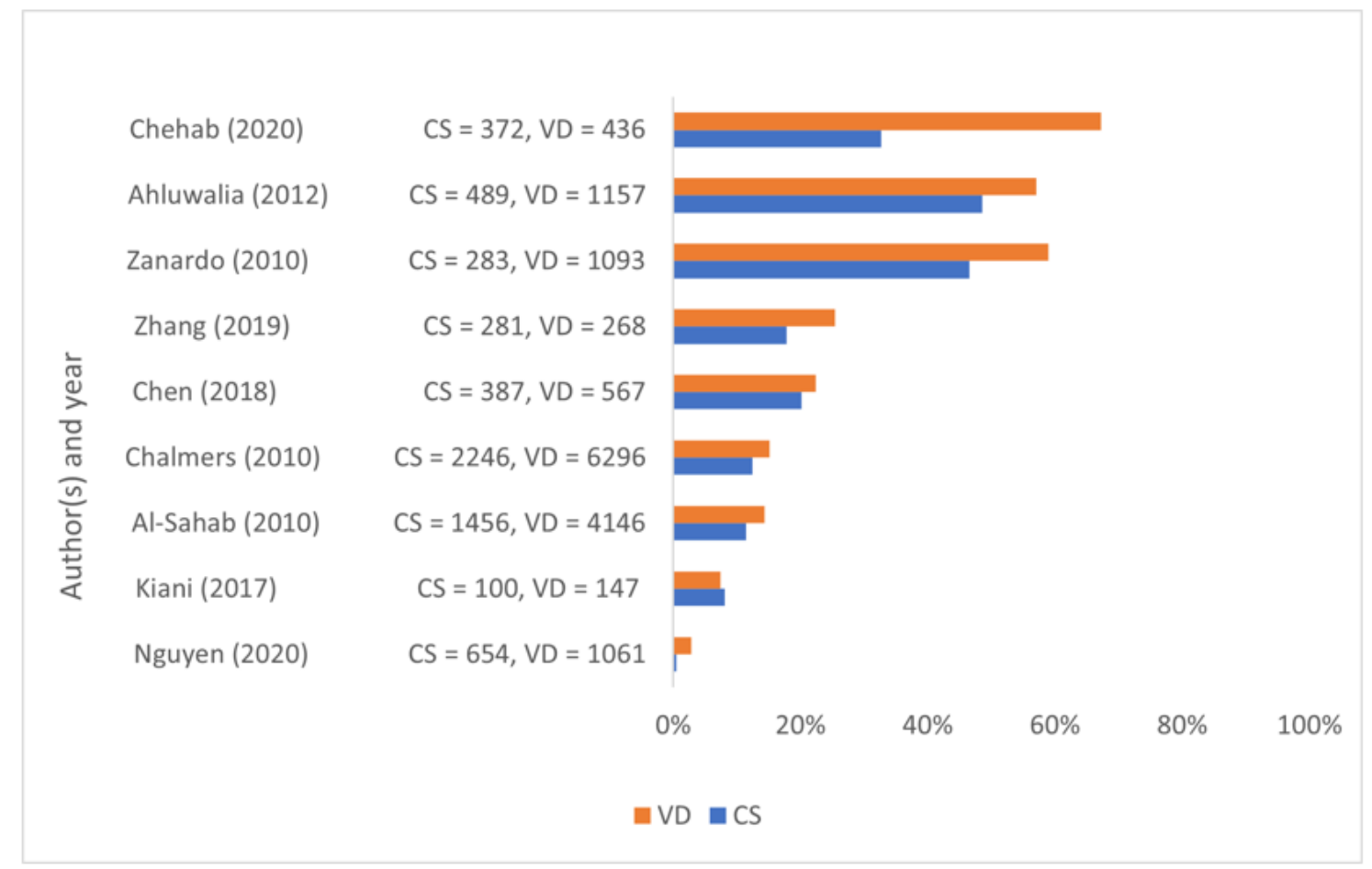

VD, Vaginal Delivery (population)

CS, C-Section (population)

Figure 7

Percentages of exclusive breastfeeding six months after delivery

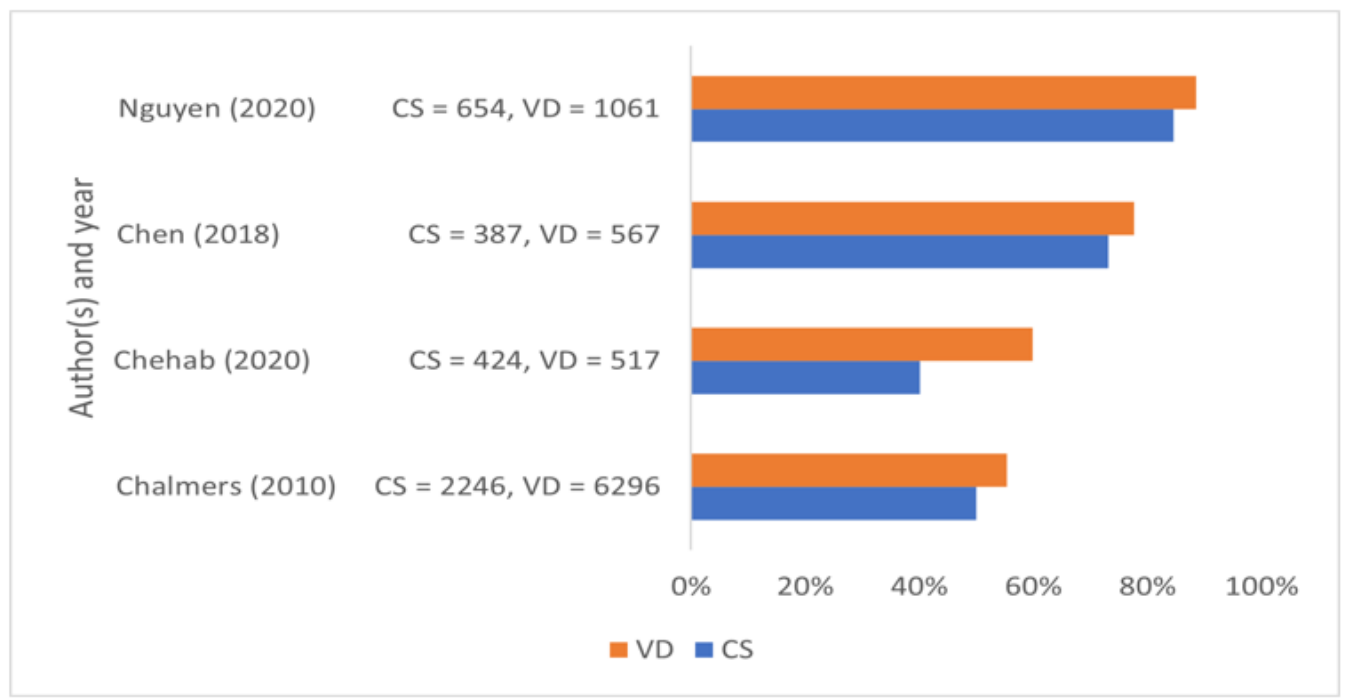

VD, Vaginal Delivery (population)

CS, C-Section (population)

\section{Figure 8}

Percentages of any breastfeeding six months after delivery

\section{Supplementary Files}

This is a list of supplementary files associated with this preprint. Click to download. 
- 2021.7.26FAppendix1.docx

Page 18/18 